\title{
Addressing androgenetic alopecia-a complex disorder-with a multilateral treatment strategy
}

\begin{abstract}
Alopecia or hair loss is a multifaceted disorder that results from influence of an array of different factors including heredity, androgenic hormones, micro-inflammation of hair follicles and scalp, infections, nutritional deficiency and harsh environmental conditions. The role of androgens and especially, the dihydrotestosterone (DHT) have been well-established as the most important players in pathophysiology of androgenetic alopecia (AGA); and elevated DHT levels have been associated with hair loss. Current treatment regimen approved by FDA includes minoxidil and finasteride. Minoxidil, administered as a topical solution is converted to minoxidil sulfate, the active metabolite by sulfotransferase enzyme at target site and hence, individual responses to treatment vary depending on the enzyme expression. Finasteride an effective $5 \alpha-$ reductase inhibitor which blocks conversion testosterone to DHT is administered orally and presents significant unwanted systemic exposure. In the present manuscript, we have presented an overview of recent advancements in our understanding of pathophysiology of AGA and have discussed underlying factors that contribute to the development/progression of the disease. Ideally due to complex nature of the disorder, a multilateral therapeutic approach addressing multiple pathophysiological pathways seems more sensible in comparison to a monophasic (single-drug) one. Moreover superior targeting of hair follicles the site of action, could be achieved with least undesired effects by if the treatments are solely administrated via topical compared with that via oral route. In later part of this review, we have focused the discussion on currently available therapeutic options for management of AGA and reviewed several emerging potential targets for development of advanced therapies.
\end{abstract}

Keywords: androgenetic alopecia, minoxidil, Finasteride, multilateral approach, $5 \alpha-$ reductase
Volume 3 Issue I - 2017

\author{
Brijeshkumar Patel,' Maria Abril Martinez \\ Velasco, 2,3 Fernando Tamez Gutierrez,' \\ Daniel Khesin' \\ 'DS Healthcare Group, Inc. Pompano Beach, USA \\ ${ }^{2}$ Hospital Medica Sur, Mexico \\ ${ }^{3}$ Universidad Nacional Autonoma de Mexico, Mexico
}

Correspondence: Brijeshkumar Patel, DS Healthcare Group, Inc. Pompano Beach, Florida, USA-33064,

Email Brijesh@DSLaboratories.com

Received: August 27, 2016 | Published: February 06, 2017
Abbreviations: AGA, androgenetic alopecia; DHT, dihydrotestosterone; FDA, food and drug administration; BFGF, basic fibroblast growth factor; IGF-1, insulin-like growth factor 1; VEGF, vascular endothelial growth factor; EMA, european medicines agency.

\section{Introduction}

Androgenetic alopecia (AGA) is a disorder that involves gradual loss of hair in genetically predisposed individuals and is more prominent in men than women. Ethnically, white male are affected the most. ${ }^{1}$ the estimated prevalence of AGA in Caucasian populations by the age of 80 is over $90 \%{ }^{2}$ Physiological changes which occur during AGA include hair follicle miniaturization and variations in normal hair cycle; specifically, anagen phase length shortens and that of telogen phase stays same or prolongs. ${ }^{3}$ In addition, the overall follicular apparatus is also gradually miniaturized which leads to global reduction of hair density due to conversion of terminal hair into vellus hair. ${ }^{4,5}$

Testosterone, a steroid hormone, has been identified as a main culprit and its presence and concentrations in hair follicles are linked to AGA occurrence; ${ }^{6}$ in addition the genetic factors have profound influence on magnitude of hair follicles response to circulating androgens. ${ }^{7}$ Also the polygenic inheritance of the disorder results from variations in the expressed phenotypes, primarily of the androgen receptor (AR) gene variants as the ARs regulates actions of androgens in hair follicles. For instance the AR polymorphism, Stu 1 is prominently associated with AGA. ${ }^{8}$ Additionally, the $5 \alpha$-reductase, aromatase and estrogen receptor genes may contribute to occurrence of AGA. ${ }^{9}$ Via the action of $5 \alpha-$ reductase, systemic testosterone is converted to dihydrotestosterone (DHT). DHT has significantly higher affinity approximately 10-fold for the receptor in comparison to testosterone. Studies show that the hair follicles of AGA-affected individuals have significantly heightened levels of both DHT and ARs. ${ }^{10}$ Upon binding of DHT to the receptors, downstream signalling pathway leads to release of specific mediators from dermal papillae causing anagen phase to end and catagen to begin in untimely manner. Such alterations in hair cycles result from reduced levels of specific factors such as IGF-1, BFGF and VEGF which are necessary to sustain anagen phase. ${ }^{11}$

Besides the adversely affecting androgens, micro-inflammation of follicular apparatus also play role in AGA manifestation. ${ }^{12}$ Activated T-cells have been observed to infiltrate near the follicular infundibulum. ${ }^{13}$ In fact the inflammation in hair follicles could result from multitude of internal and/or external factors such as due to microbial infection, use of harsh chemicals, oxidative stress, normal aging, smoking and excessive exposure to sunlight and pollutants. Each of these factors are capable of increasing levels of free radicals in hair follicles which is detrimental to overall follicle health.

More recently, several studies have revealed the important role of prostaglandins (PGs) in governing the hair cycle. Prostaglandins are lipidic mediators synthesized via arachidonic acid pathway under the actions of specific enzymes. Amongst investigated PGs, PGD2, PGE2, and PGF2a have exhibited activities related to governing 
hair cycle. More specifically studies have demonstrated hair growth inhibitory effects of PGD2, whereas hair growth stimulating effects of PGE2 and PGF2a. ${ }^{14,15}$ Increased levels of PGD2 and reduced levels of PGE2 have been observed in AGA affected male scalp. ${ }^{14}$ Amongst the two receptors of PGD2, GPR44 and PTGDR, ${ }^{16}$ the former expresses predominantly in hair follicles and upon association with the ligand, may trigger follicle miniaturization by interfering stem cell maturation and thwarting development of terminal hair from vellus hair. ${ }^{16}$

Overall based on currently available scientific information, it is evident that an array of different factors-dependent or independent, physiological or external-play role in progression of AGA and hence, the exact pathogenetic mechanism underling AGA is complex and poorly understood. Moreover, as AGA results from influence of one or more underlining factors and the individual contributions in overall progression of AGA of these factors varies person-to-person, it sounds scientifically prudent to address the issue with a treatment that targets multiple underlining factors/pathways. In the present review, we have summarized recent advancements in our understanding of pathophysiology of AGA, discussed currently available therapeutic options for management of AGA and presented an overview of emerging potential targets for development of advanced therapies.

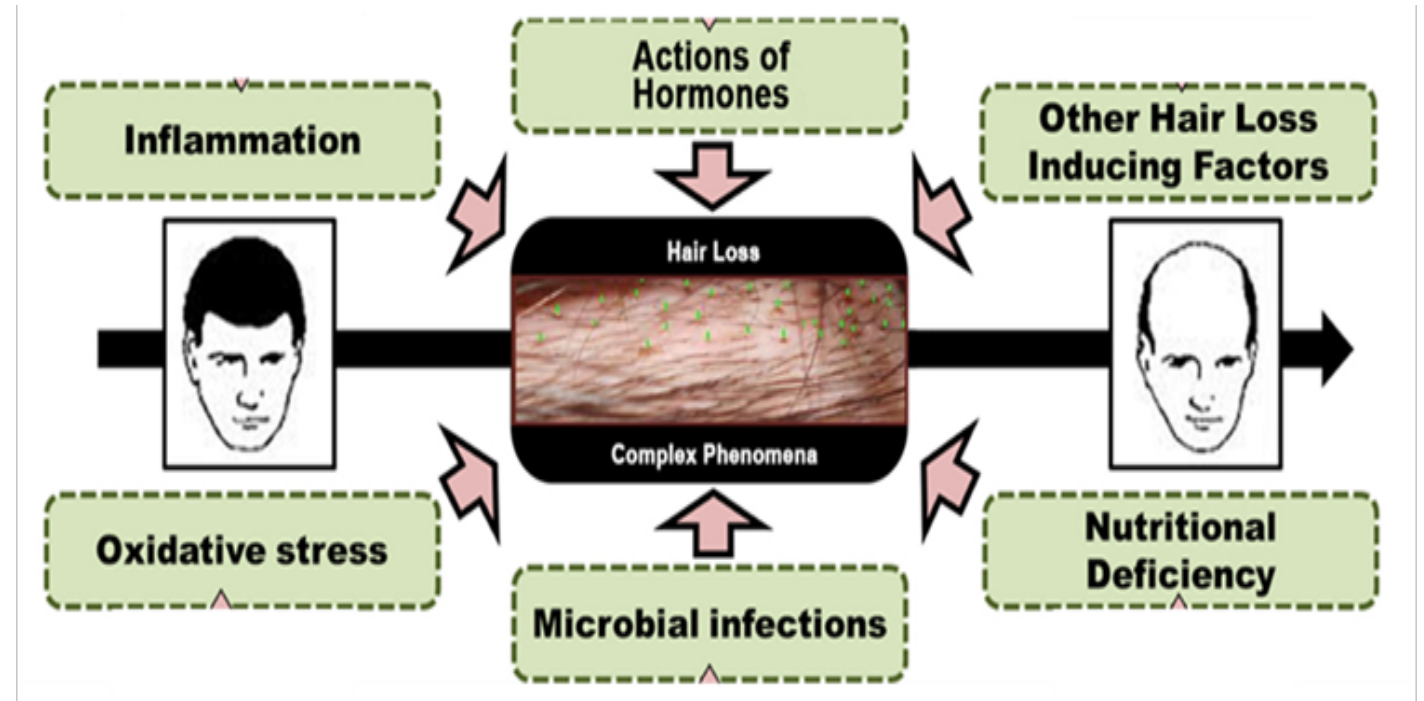

Figure I Factors involved in pathophysiology of alopecia.

\section{Current strategies for management of AGA}

Physiologically androgenetic alopecia is a multilateral phenomenon; it causes considerable changes in overall appearance of patients and is associated with enormous subsequent psychological and social implications, especially in younger men. ${ }^{1}$ Although it is well accepted that several pathogenic mechanisms are involved in the onset of AGA, only two pharmaceutical interventions are currently available. A topical solution and an oral drug with systemic exposure are available for use either individually or together depending on severity of AGA, individual response to treatments, affordability and risks. ${ }^{17}$ Till date minoxidil topical solution and finasteride, in form of oral tablets, are the only two therapeutic options approved by the United States Food and Drug Administration (US-FDA) and European Medicines Agency (EMA) for treatment of AGA. ${ }^{5}$

\section{Minoxidil}

Topically administered Minoxidil, available in forms of $2 \%$ and $5 \%$ solution and foam is the most widely recommended drug ${ }^{18}$ as the first-line therapy in case of mild to moderate androgenetic alopecia. ${ }^{19}$ Although the exact mechanism of action of minoxidil is not fully elucidated, the hair growth stimulating actions are attributed to its active metabolite, minoxidil sulphate, which produces vasodilatation in peripheral arteries by activating ATP-dependent potassium channels in vascular smooth muscles. Minoxidil is also reported to promote angiogenesis by enhancing VEGF mRNA expression in dermal papilla. ${ }^{20}$ In in vitro settings, minoxidil stimulates PGE2 and leukotriene B4 expression, and block prostacyclin production in by dermal papilla cells. Also the expression of EP2, one of the PGE2 receptors responsible for lengthening of anagen phase is amplified under the influence of minoxidil treatment. Further, due to antifibrotic activity of minoxidil inhibition of enzyme lysyl hydroxylase present in fibroblast may result in synthesis of a hydroxylysinedeficient collagen. Owing to the effects of minoxidil treatment, the miniaturized hair follicle stays longer in the anagen phase, decreasing shedding and producing hair similar to the terminal hair. ${ }^{21}$

Pharmaceutically, commercial minoxidil solutions use ethyl alcohol, propylene glycol and water as solvents to solubilize minoxidil for its topical delivery. Currently, minoxidil is approved by FDA in form of a solution and a foam, both in strengths of $2 \%$ and $5 \% \mathrm{w} / \mathrm{v}$. The hair growth promoting efficacy of minoxidil is strengthdependent. ${ }^{22}$ For maximum results, consumers are instructed to apply $1 \mathrm{ml}$ minoxidil solution two times per day at least for 4-6 months. Side effects associated with minoxidil treatment are limited, mainly related to scalp pruritus, irritation and scaling. In addition, consumers have also reported higher incident of itching, redness and drying scalp with 5\% minoxidil solution. ${ }^{1}$ In the beginning of minoxidil treatment, initial shedding of hair has also been commonly experienced due to molting of telogen hair. ${ }^{17}$ In certain cases, hair growth can also be observed in areas other than scalp due to systemic absorption of topically applied minoxidil. ${ }^{22}$

Several studies have investigated effects of combining other active ingredients with minoxidil with an objective of achieving superior outcomes for the treatment of AGA. In comparison to minoxidil alone, its combination with pyrithione zinc or hydrocortisone have shown superior hair growth. ${ }^{23}$ In this clinical study, patients observed faster prevention of hair loss and appearance of newly grown hair after 
treatment with Minoxidil plus Adenosine in comparison to Minoxidil alone. ${ }^{24}$ In a separate clinical study, minoxidil topical application produced significant increase in hair density and thickness; and treatment with alfatradiol resulted in deceleration or stabilization of hair loss. ${ }^{25}$ In another study, the effects of Minoxidil high extra combination (MHEC) containing 12.5\% Minoxidil, 5\% Azelaic acid and $0.025 \%$ Betamethasone on hair growth were investigated and compared with Minoxidil 5\% in a randomized controlled trial of 116 patients with androgenic alopecia. MHEC showed significant reduction in hair shedding compared to Minoxidil 5\% alone. Further, the patients tolerated MHEC without significant adverse effects in comparison to placebo or Minoxidil 5\% alone. ${ }^{26}$

\section{Finasteride}

Finasteride a selective type II $5 \alpha$-reductase inhibitor is the first FDA-approved oral treatment for AGA being sold under brand name Propecia ${ }^{\circledR}$. The mechanism of action of finasteride involves its irreversible binding with enzyme and blocking production of DHT from testosterone; ${ }^{27}$ thus causing reduction in DHT-induced follicular miniaturization. In a long-term (5 year) controlled efficacy study, finasteride produced significant reduction in hair loss at a dose of $1 \mathrm{mg}$ per day in human subjects with AGA. In another 10 year-long study, finasteride administered orally at $1 \mathrm{mg}$ daily dose was observed to be safe over longer period of time with pronounced and sustained hair growth in male subjects. ${ }^{28}$ Also, few of the enrolled male subjects were noticed to be non-responsive to the long term treatment based on lack of or minimum improvements observed in hair growth during the first year of finasteride treatment. ${ }^{28}$

At a dose of $1 \mathrm{mg}$ per day, adverse effects associated with finasteride therapy are limited, mainly related to sexual functions which are expected to go away after discontinuation of the therapy. ${ }^{29}$ The key enzymes responsible for precipitating these adverse effects are aromatase, which increases production of estradiol from testosterone, and $5 \alpha$-reductase which reduces production of DHT from testosterone. Increased estradiol has been linked to specific side effects such as feminization and gynecomastia; whereas, reduced DHT may result in loss of libido, erective dysfunction and other sexual side effects. Although the incidents of such adverse effects are less, this may affect overall compliance of patients to this therapy. ${ }^{28}$

The major shortcoming associated with finasteride is its approved route of delivery. The site of action for finasteride is at hair follicles located on scalp; however, the current FDA-approved formulation is a tablet for an oral administration, which causes unwanted exposure of drug to systemic circulation and to other organs and in turn increases the risk of producing several undesired adverse effects. In order to avoid such side effects, many of the research groups have theorized and explored the strategy of administering finasteride directly to the scalp through topical delivery. ${ }^{29,30}$ In fact, in a shortterm study topical finasteride solution $(0.25 \%)$, with significantly lesser systemic exposure, generated almost same inhibitory effects on plasma DHT levels as that observed with $1 \mathrm{mg}$ per day oral finasteride administration. ${ }^{30}$ In a separate intermediate-term study, topical gel containing $1 \%$ finasteride was found to be as efficacious as $1 \mathrm{mg}$ per day oral finasteride. ${ }^{31}$ In addition, the approach of delivering finasteride via topical route opens up several new possibilities such as combining topical minoxidil with topical finasteride which may improve overall efficacy, reduce potential health risks and provide superior patient compliance. In fact in a pioneering study, compared with minoxidil alone, topical minoxidil and finasteride combination was observed to be more efficacious in promoting hair growth. ${ }^{32}$
Taking all together, till date only topical minoxidil and oral finasteride are the only approved therapies by FDA for AGA. Considering the multitude of factors involved in causing the disorder, therapies other than just minoxidil and finasteride are needed to be explored and developed. In the subsequent section, we have discussed several new chemical compounds and agents from botanical origins which have shown significant potential in recent years in addressing AGA.

\section{Emerging agents with potential in treatment of AGA}

\section{Alfatradiol}

Alfatradiol (also called $17 \alpha$-estradiol) is a stereoisomer of female hormone $17 \beta$-estradiol and is commercially available in Europe and South America for the treatment of AGA in women. Several mechanisms have been proposed for actions of estrogens in mediating their beneficial effects on AGA-affected hair follicles. Ex vivo study using hair follicles, showed increased rate of conversion of testosterone to estradiol upon treatment with Alfatradiol owing to its aromatase stimulating activity ${ }^{33}$ and thus, decreasing the level of testosterone and eventually of DHT. Further, in an in vitro experiment alfatradiol stimulated the growth of human hair bulb papilla cells and hair root sheath fibroblast. ${ }^{34}$ In a clinical trial, topical application of alfatradiol solution exhibited statistically significant increase in number of hair and diameter from baseline; and hair improvement in self-assessment and global photographic assessment compared with placebo treatment. ${ }^{35}$ Additionally in a separate clinical study, alfatradiol after topical application showed reduction or stabilization of hair loss. ${ }^{36}$

\section{In vitro-reductase inhibitors}

Dutasteride: Although it is currently approved for the treatment of benign prostate hyperplasia, Dutasteride, a dual inhibitor of both type I and type II $5 \alpha$-reductase commercially sold with brand name Avodart $^{\circledR}$ has shown superior hair growth stimulating effects than that of finasteride ${ }^{37}$ is the predominant subtype of enzyme present in hair follicles; whereas, $5 \alpha$-reductase type I is responsible for regulating sebum production and is constitutively present in sebum glands. Dutasteride at lower concentration of $0.5 \mathrm{mg} / \mathrm{d}$ has shown higher inhibition of type II $5 \alpha$-reductase in comparison to finasteride $(1 \mathrm{mg} / \mathrm{d})$, pointing out its enhanced efficacy in reducing DHT levels. A multicenter, prospective human clinical study in Japan observed that dutasteride is safe, well-tolerated and efficacious over the period of 52 weeks at daily dose of $0.5 \mathrm{mg}$ in AGA-affected male subjects. ${ }^{38}$ Also in this placebo controlled study dutasteride treatment significantly increased hair count. Taking together due to its dose-dependent efficacy in reducing DHT blocking type II $5 \alpha-$ reductase and inhibitory actions on sebum production owing to blockage of type I $5 \alpha-$ reductase, dutasteride is assumed to have significant potential to be developed as the next therapeutic agent for AGA.

$\boldsymbol{\beta}$-sitosterol: Agents from plant sources are gaining enormous significance in recent times due to perceived advantages such as lack or minimum of associated side effects in comparison to drugs. Several of the plant extract with proposed inhibitory action on $5 \alpha$-reductase enzyme have been investigated in the treatment of benign prostate hyperplasia. Few of them with potentially beneficial effects in addressing AGA are $\beta$-sitosterol and Canadian willow herb extract. Saw palmetto (Latin: Serenoa repens) extract and its major constituent, $\beta$-sitosterol have been in past used for relief in benign prostatic 
hyperplasia treatment. Recent studies indicate that it may have potential in the treatment of less severe cases of AGA. ${ }^{19}$ Constituents of saw palmetto extract have been reported to possess anti-androgenic activities by competitively blocking both type I and II $5 \alpha-$ reductase in indiscriminately manner. ${ }^{39,40}$ This results in reduction in DHT influence on hair follicles. In addition, via estrogen receptor-mediated actions saw palmetto assists in maintenance and prolongation and of anagen phase. Supplementation with saw palmetto, via oral route, has shown beneficial effects in male subjects suffering from AGA. ${ }^{39}$ Also, in a comparative study saw palmetto extract induced positive hair growth in $38 \%$ subjects in comparison to the control finasteride treated group, where improvements in hair growth were observed in $68 \%$ subjects. ${ }^{40}$ Interestingly, the hair growth promoting effects of saw palmetto were limited to only vertex, whereas, both frontal and vertex areas showed improvements after finasteride treatment. ${ }^{40}$ Saw palmetto extract seemed to be well-tolerated in human subjects and the only adverse effect observed is abdominal discomfort. Advantageously, saw palmetto extract does not influence prostate-specific antigen levels or obstruct in any diagnostic procedures, which is the case with finasteride treatment.

Canadian willow herb: Epilobium a member of the Onagraceae family is a perennial herb widely distributed all over the world; it consists of over 200 species that tolerate a wide range of site and soil conditions. In past, Epilobium species have used as a preventative measure for benign prostatic hyperplasia (BPH) $;^{41-44}$ and many reports suggest of plant possessing anti-proliferative, anti-inflammatory and antioxidant activities. ${ }^{45-52}$ More specifically, Epilobium extracts have shown noticeable effects on activities of several key enzymes including $5 \alpha-$ reductase and aromatase,,$^{53,54}$ caspase $3^{55}$ and metaloproteinases. ${ }^{56}$

In addition to anti-androgenic properties, Epilobium extracts also possess anti-inflammatory and prostaglandin inhibition properties mainly due to presence of myricetin $3-\mathrm{O}-\beta-\mathrm{D}-$ glucuronide which hinders the PGI2, PGE2, and PGD2 release. A study reported oenothein $\mathrm{B}$, the tannin from various fireweeds, to be the active compound inhibiting $5 \alpha$-reductase in the human prostate. ${ }^{49}$ This is also supported by the observation that Epilobium herbal extract stimulated activity of estrogen $\alpha$ receptor and inhibited that of estrogen $\beta$ receptor in rats in response to externally administered testosterone..$^{57}$

Ducrey et al. ${ }^{53}$ reported the active compounds of Epilobium extract, oenothein A and B inhibiting $5 \alpha-$ reductase and aromatase enzymes. The oral administration of standardized Epilobium extract produced significant reduction in 5 $\alpha$-reductase type II mRNA expression in a murine model; and noticeably, the observed reduction in enzyme mRNA expression with Epilobium treatment was more pronounced than that seen after finasteride treatment. ${ }^{58}$ In a controlled clinical trial, once-a-day Epilobium extract treatment significantly reduced international prostate specific score in male subjects in comparison to placebo treatment over 3 months. ${ }^{59}$ Overall, as a botanical source for a novel treatment for AGA, Epilobium species have shown significant potential and warrant further exploration owing to their potent $5 \alpha-$ reductase type II and PGD2 inhibiting activities.

Fluridil: Based on recent advancement in our understanding of role of androgen receptors in progression of AGA, a strategy of using androgen receptor blockers seems sensible in AGA treatment. ${ }^{7}$ The main challenge associated with this approach is to develop an agent that is devoid of concerns of systemic effects, especially in male which could result in feminization, gynecomastia and impotence, and specifically targets androgen receptors in the hair follicles. Fluridil an analog of flutamide is a recently developed agent specifically for only topical administration; it presents anti-androgenic activities when delivered directly to the hair follicles and if absorbed systemically breakdowns into inactive metabolites. In an only clinical study published, fluridil treatment exhibited significant improvements in anagen/telogen hair ratio within 90 days in comparison to vehicle (placebo) treatment; and, no adverse effects related to sexual performance or blood chemistry were observed. ${ }^{1,60-62}$

\section{Ant-inflammatory, antioxidant and antimi- crobial agents}

\section{Ketoconazole}

Although it is a broad-spectrum anti-fungal agent, ketoconazole is observed to impede synthesis of steroid at high concentrations through inhibition of cytochrome P450 and lyase. ${ }^{17,20}$ Ketoconazole topical treatment was observed to promote hair growth by increasing density and size of anagen follicles when used over period of 21 weeks at a concentration of $2 \%$ in a shampoo formulation. Though anti-DHT activities of ketoconazole have been reported, the major beneficial effects were suggested to be arising from its inhibitory actions on inflammation and fungal infections in the scalp. ${ }^{63}$ Also in a murine model, $2 \%$ ketoconazole topical treatment observed to stimulate hair growth. ${ }^{64}$ In a human clinical study, treatment with ketoconazole $1 \%$ shampoo resulted in increased anagen hair percentage and hair shaft diameter along with reduction in hair shedding, telogen effluvium and scalp dandruff. ${ }^{65}$ Taking all together, ketoconazole has significant potential to be developed as a stand-alone or an adjunct therapy in combination with existing therapeutic options for the treatment of AGA depending on the severity of disease in an individual.

\section{Prostaglandin F2a analogues}

PGs influence follicular function and hair cycle and hence, present themselves as a potential target for development of future AGA therapies..$^{14,16}$ A recent study has reported increased levels of both PGD2 synthase and its mRNA in AGA affected scalp in comparison to healthy ones, causing regression and inhibition of hair growth. ${ }^{66}$ Prostaglandin analogs, namely bimatoprost and latanoprost are FDA-approved drugs indicated for reducing intra-ocular pressure in glaucoma patients; however serendipitously, these agents were observed to induce increase in both length and number of eyelashes. ${ }^{15}$ This led to bimatoprost receiving FDA approval for eyelash hypotrichosis following extensive clinical studies.$^{65}$ In a first of a kind in vivo animal study, latanoprost exhibited noticeable hair growth promoting activities in comparison to vehicle-treated group.$^{66}$ The hair growth stimulating effects of prostaglandin analogs are postulated to be due to elongation of anagen phase; in fact, prostaglandins could be mediating minoxidil induced hair growth as minoxidil was suggested to promote production of PGs.

\section{Melatonin}

Besides testosterone and DHT other hormone that affects hair growth and regulates hair cycle is melatonin an endogenous molecule secreted by pineal gland. Melatonin has been observed in several species, influence production and timings of wool and cashmere and even the color of hair coat. ${ }^{67}$ Although the role of melatonin on hair biology is not fully understood, it is considered to be very complex as hair related activities of melatonin different significantly depending dose, gender and species. Melatonin does not only interact with 
specific receptors but also influence androgen and estrogen receptormediated downstream processes. It also inhibits both apoptosis and expression of estrogen receptor in mice skin. Further, through its free radical scavenging and protective actions, melatonin may assist anagen hair bulb in maintaining its high proliferative and metabolic activities. On the other hand, melatonin may induce nuclear exclusion of androgen receptor and thus, produce hair growth inhibitory effects. ${ }^{68}$ Such melatonin-mediated nuclear exclusion of ARs occurs via activation of calcium and protein kinase $\mathrm{C}$ pathway. ${ }^{69} \mathrm{In}$ a clinical study on conducted on female patients suffering from diffuse alopecia and AGA, hair growth promoting efficacy of twice-a-day melatonin $0.1 \%$ topical solution was evaluated. ${ }^{70}$ Over the period of 6 months, patients with AGA showed significant improvements in number of anagen hair in occipital region, almost twice the number of anagen hair to that of non-anagen hair in comparison to placebo treatment.

\section{Cetirizine}

PGD2, via its actions on GPR44 receptor located in follicles influences hair growth. In fact, the expression of GPR44 has been observed to be higher in hair follicles of AGA affected patients. ${ }^{14}$ Considering this as a new therapeutic target, agents capable of inhibiting PGD2 association with its receptor could be of great interest as a novel therapy for androgenetic alopecia. Cetirizine a second generation anti-histaminic agent and more specifically an $\mathrm{H} 1$ receptor blocker has been observed to reduce PGD2 release from mast cells, ${ }^{71}$ whereas, increase the release of PGE2 in a concentrationdependent manner, from human monocytes in in vitro setting. ${ }^{72}$ Based on this observations another study designed to evaluate the efficacy of topical $1 \%$ cetirizine solution reported significant improvement in hair growth in patients with mild to moderate AGA. ${ }^{73}$ In addition, the anti-inflammatory and anti-allergic activities of cetirizine can be useful in AGA patients with scalp irritation and inflammation.

\section{Nutritional deficiencies}

\section{Vitamin D}

The Vitamin D receptor (VDR), which has a significant influence on epidermal homeostasis in ligand-dependent manner controls epidermal keratinocyte proliferation and differentiation. VDR expression has been linked to reduced proliferation and heightened differentiation of keratinocytes which assist in efficient hair cycle progression. ${ }^{72}$ Expression of VDR is of more significance for the keratinocyte stem cells located in follicular bulge as any dysfunctionalities in VDR may result in hair cycle progression defects. ${ }^{74}$ In fact, the expression of VDR in keratinocytes has been associated with and varies depending on the specific stage of the hair cycle the follicle is in. ${ }^{18}$ In an in vivo study using mouse model, although topical calcitriol pretreatment was observed to be ineffective in preventing chemotherapy-induced alopecia; the pretreatment was beneficial in terms of speedy recovery and superior regrowth of hair following drug-induced hair loss. Interestingly, the improvements were global and not limited to the site of administration, indicating the role of calcitriol pretreatment in quick reconstruction of anagen hair follicles. ${ }^{75}$ Unlike stated earlier, another study observed protective efficacy of calcitriol following topical administration in paclitaxel and cyclophosphamide-induced alopecia. ${ }^{76}$ While the actions of vitamin D have not been thoroughly explored in human hair cycle processes, it has gain significant attention of researchers as either a preventative or reactive treatment in chemotherapy-induced alopecia.

\section{Zinc}

Zinc one of the essential trace elements, has substantial role on almost all aspects of metabolic reactions occurring in biological organs, including skin. ${ }^{77}$ Specifically, Zinc may influence hair biology via its immunomodulatory effects. ${ }^{77,78}$ Its antioxidant effects may stem from indirect stimulation of certain endogenous chemicals into metallothionein which possess superior antioxidant activities. ${ }^{79}$ Biologically through its ability to inhibit endonucleases, Zinc may slow down the apoptotic processes in the hair follicles, specifically in keratinocytes during regression phase of the hair cycle catagen. In fact, such inhibitory effects of Zinc on endonuclease enzymes may be of great importance in preventing follicle deterioration..$^{80}$ In addition, zinc is necessary for maintaining stable DNA and for its repair; given that the rate of proliferation of follicular apparatus, and more importantly the associated epithelia cells is very high sustained and proper availability of zinc is necessary for its efficient maintenance. ${ }^{81}$ Zinc deficiency has been linked to several undesired hair and scalp conditions such as telogen effluvium, weaker hair and nail, seborrhea, dermatitis, and scalp infections. ${ }^{82}$ Microscopic observations have revealed certain histological changes such as yellowing of epidermal cells, single-cell necrosis, thinning of hair shafts ${ }^{83}$ and asymmetrical striations. ${ }^{84}$ However, it is very important to supervise the zinc levels during supplementary therapy as over-consumption of zinc is associated with certain adverse effects such as drowsiness, headache and deficiency of some other essential elements.

\section{Radiation-induced alopecia}

Radiation therapy is commonly used alone or in conjunction with chemotherapeutic agents or surgery for the treatment of certain types of cancer. Notably, the radiation-induced hair loss is often observed in patients exposed to high-levels of radiation. ${ }^{85}$ Owing to the higher frequency of precipitation of such radiation-induced alopecia, patients under stress of losing hair may consider declining the treatment. Alopecia resulting as an adverse reaction of radiation treatment not only leads to superficial cosmetic concerns but may also induce more serious social and psychological issues in patients; and hence, it is imperative that patients clearly understand the probable outcomes, related to hair loss of the treatment and have expectations of prevention or reversal of hair loss during the radiation therapy. In fact, several research groups are investigating potential ways of preventing and/or inducing quick hair regrowth following radiation treatment in patients. In a murine model, the protective effects of a PGE2 analog was investigated in preventing radiation-alopecia, where 16,16-dimethyl PGE2, when administered either topically or subcutaneously, assisted in retaining hair counts to a notable extent in the target area following $137 \mathrm{Cs}$ gamma irradiation. ${ }^{86}$ In another study, topical pretreatment of nitroxide, either Tempo or Tempol was investigated in guinea pigs for their radio-protective effects following multi-dose radiation therapy. Although both controlled untreated and treated animals showed gradual loss of hair over 14 weeks post radiation therapy, the hair loss was significantly less prominent in nitroxide treated animals in comparison to untreated group. Further, the observed hair density in Tempo-treated animals was around $90 \%$ of that observed before irradiation, whereas untreated animals showed hair density of only $12 \%$ over weeks 11 to 14 . The study concluded that the nitroxide based protective agents could be of high clinical importance in combating radiation-induced alopecia. ${ }^{87}$ Notably, radiation dose and the frequency/depth of exposure are observed to be key factors in influencing the extent of radiation-induced alopecia; 
in fact, a study reported that if the radiation dose is kept under 16 Gy with superficial exposure to a depth of $5 \mathrm{~mm}$, the probability of cicatricial alopecia is significantly reduced after radiation therapy. ${ }^{88}$ Another approach to address radiation-induced alopecia, which is under investigation is employing punch graft technique of hair transplant with modified approach of selecting donor and recipient areas. ${ }^{89,90}$

\section{Conclusion}

Multilateral therapeutic approach has been gaining significant attention in case diseases which result from more than one underlining cause contributing simultaneously. As it has established that the occurrence and advancement of androgenetic alopecia involve multitude of confounding factors, addressing the complex condition with only available therapeutic options, minoxidil and finasteride may not be the most efficient strategy. In addition, the shortcoming with these approved therapies also need to be considered; individual response to minoxidil may vary person-to-person owing to differences in expression of sulfotransferase enzyme, whereas, oral administration of finasteride produces unwanted systemic exposure of drug and theoretically poses risk of adverse effects. As discussed in this review, recent advancements towards investigating and identifying novel therapeutic targets in pathology of AGA have revealed several new compounds with substantial potential in emerging as an innovative breakthrough for the treatment of alopecia.

\section{Acknowledgements}

None.

\section{Conflict of interest}

The author declares no conflict of interest.

\section{References}

1. Rathnayake D, Sinclair R. Male androgenetic alopecia. Expert Opin Pharmacother. 2010;11(8):1295-1304.

2. Hamilton JB. Patterned loss of hair in man:types and incidence. Ann NY Acad Sci. 1951;53(3):708-728.

3. Guarrera M, Rebora A. Anagen Hairs May Fail to Replace Telogen Hairs in arly Androgenic Female Alopecia. Dermatology. 1996;192(1):28-31.

4. Rousso DE, Kim SW. A review of medical and surgical treatment options for androgenetic alopecia. JAMA facial Plast Surg. 2014;16(6):444-450.

5. Banka N, Bunagan MK, Shapiro J. Pattern hair loss in men:diagnosis and medical treatment. Dermatol Clin. 2012;31(1):129-140.

6. Ellis JA, Sinclair RD. Male pattern baldness:current treatments, future prospects. Drug Discover Today. 2008;1(17-18):791-797.

7. Ellis JA, Sinclair R, Harrap SB. Androgenetic alopecia:pathogenesis and potential for therapy. Expert Rev Mol Med. 2002;4(22):1-11.

8. Hillmer AM, Hanneken S, Ritzmann S, et al. Genetic variation in the human androgen receptor gene is the major determinant of common early-onset androgenetic alopecia. Am J Hum Genet. 2005;77(1):140-148.

9. Ellis A, Harrap SB. The genetics of androgenetic alopecia. Clin Dermatol. 2001;19:149-154.

10. Randall VA. Androgens and human hair growth. Clin Endocrinol (oxf) 1994;40(4):439-457.

11. Thornton MJ, Hamada K, Randall VA, et al. Androgen-dependent beard dermal papilla cells secrete autocrine growth factor (s) in response to testosterone unlike scalp cells. J Invest Dermatol. 1998;111(5):727-732.
12. Whiting DA. Diagnostic and predictive value of horizontal sections of scalp biopsy specimens in male pattern androgenetic alopecia. $J \mathrm{Am}$ Acad Dermatol. 1993;28(5 pt 1):755-763.

13. Mahe YF, Michelet JF, Billoni N, et al. Androgenetic alopecia and microinflammation. Int J Dermato. 2000;39(8):576-584.

14. Garza LA, Liu Y, Yang Z, et al. Prostaglandin D2 inhibits hair growth and is elevated in bald scalp of men with androgenetic alopecia. Sci Transl Med. 2012;4(126):126ra134.

15. Johnstone MA, Albert DM. Prostaglandin-induced hair growth. Surv Ophthalmol. 2002;47(Suppl 1):S185-S202.

16. Nieves A, Garza LA. Does prostaglandin D2 hold the cure to male pattern baldness? Exp Dermatol. 2014;23(4):224-227.

17. Blumeyer A, Tosti A, Messenger A, et al. Evidence-based (S3) guideline for the treatment of androgenetic alopecia in women and in men. J Dtsch Dermatol Ges. 2011;9(Suppl 6):S1-S57.

18. Bienova M, Kucerova R, Fiuraskova M, et al. Androgenetic alopecia and current methods of treatment. Acta Dermatovenerol Alp Pannonica Adriat. 2005;14(1):5-8.

19. Rossi A, Calvieri S. Treatment for alopecia. G Ital Dermatol Venereol. 2014;149:103-106.

20. Lachgar S, Charveron M, Gall Y, et al. Minoxidil upregulates the expression of vascular endothelial growth factor in human hair dermal papilla cells. Br J Dermatol. 1998;138(3):407-411.

21. Rossi A, Cantisani C, Melis L, et al. Minoxidil use in dermatology, side effects and recent patents. Recent Pat Inflamm Allergy Drug Discover. 2012;6(2):130-136

22. Kaliyadan F, Nambiar A, Vijayaraghavan S. Androgenetic alopecia:an update. Indian J Dermatol Venereol Leprol. 2013;79(5):613-625.

23. Gupta A, Charrette A. Topical Minoxidil:Systematic Review and Meta-Analysis of Its Efficacy in Androgenetic Alopecia. Skinmed. 2015;13(3):185-189.

24. Faghihi G, Iraji F, Rajaee Harandi M, et al. Comparison of the efficacy of topical minoxidil $5 \%$ and adenosine $0.75 \%$ solutions on male androgenetic alopecia and measuring the patients' satisfactory rate. Acta Dermatovenerol Croat. 2013;21(3):155-159.

25. Blume-Peytavi U, Kunte C, Krisp A, et al. Comparison of the efficacy and safety of topical minoxidil and topical alfatradiol in the treatment of androgenetic alopecia in women. J Dtsch Dermatol Ges. 2007;5(5):391395.

26. Pazoki-Toroudi H, Babakoohi S, Nilforoushzadeh M, et al. Therapeutic effects of minoxidil high extra combination therapy in patients with androgenetic alopecia. Skinmed. 2012;10(5):276-282.

27. Drake L, Hordinsky M, Fiedler V, et al. The effects of finasteride on scalp skin and serum androgen levels in men with androgenetic alopecia. $J$ Am Acad Dermatol. 1999;41(4):550-554.

28. Rossi A, Cantisani C, Scarno M, et al. Finasteride, $1 \mathrm{mg}$ daily administration on male androgenetic alopecia in different age groups:10-year follow-up. Dermatol Ther. 2011;24(4):455-461.

29. Mysore V, Shashikumar B. Guidelines on the use of finasteride in androgenetic alopecia. Indian J Dermatol Venereol Leprol. 2016;82(2):128134.

30. Caserini M, Radicioni M, Leuratti C, et al. Effects of a novel finasteride $0.25 \%$ topical solution on scalp and serum dihydrotestosterone in healthy men with androgenetic alopecia. Int J Clin Pharmacol Ther. 2016;54(1):19-27.

31. Hajheydari Z, Akbari J, Saeedi M, et al. Comparing the therapeutic effects of finasteride gel and tablet in treatment of the androgenetic alopecia. Indian J Dermatol Venereol Leprol. 2009;75(1):47-51. 
32. Tanglertsampan C. Efficacy and safety of 3\% minoxidil versus combined $3 \%$ minoxidil $/ 0.1 \%$ finasteride in male pattern hair loss:a randomized, double-blind, comparative study. J Med Assoc Thai. 2012;95(10):13121316

33. Hoffmann R, Niiyama S, Huth A, et al. 17alpha-estradiol induces aromatase activity in intact human anagen hair follicles ex vivo. Exp Dermatol. 2002;11(4):376-380.

34. Arai A, Von Hintzenstern J, Kiesewetter F, et al. In vitro effects of testosterone, dihydrotestosterone and estradiol on cell growth of human hair bulb papilla cells and hair root sheath fibroblasts. Acta Derm Venereol. 1990;70(4):338-341.

35. Kim JH, Lee SY, Lee HJ, et al. The Efficacy and Safety of 17alpha-Estradiol (Ell-Cranell(R) alpha $0.025 \%$ ) Solution on Female Pattern Hair Loss:Single Center, Open-Label, Non-Comparative, Phase IV Study. Ann Dermatol. 2012;24(3):295-305.

36. Olsen EA, Hordinsky M, Whiting D, et al. The importance of dual $5 \alpha-$ reductase inhibition in the treatment of male pattern hair loss:results of a randomized placebo-controlled study of dutasteride versus finasteride. $J$ Am Acad Dermatol. 2006;55(6):1014-1023.

37. Tsunemi Y, Irisawa R, Yoshiie H, et al. Long-term safety and efficacy of dutasteride in the treatment of male patients with androgenetic alopecia. J Dermatol. 2016;43(9):1051-1058

38. Prager N, Bickett K, French N, et al. A randomized double-blind, placebo-controlled trial to determine the effectiveness of botanically derived inhibitors of $5-\alpha-$ reductase in the treatment of androgenetic alopecia. $J$ Altern Complement Med. 2002;8(2):143-152.

39. Rossi A, Mari E, Scarno M, et al. Comparitive Effectiveness and Finasteride Vs Serenoa Repens in Male Androgenetic Alopecia:A Two-Year Study. Int J Immunopathol Pharmacol. 2012;25(4):1167-1173.

40. Van Hellemont J. Compendium de Phytotherapie. Belgium: Bruxelles, Belge, Association Pharmaceutique; 1986.

41. Wichtl M. Herbal drugs and phytopharmaceuticals:a handbook for practice on a scientific basis. Medpharm CRC press; 2004. 708 p.

42. Hevesi B, Houghton P, Habtemariam S, et al. Antioxidant and antiinflammatory effect of Epilobium parviflorum Schreb. Phytother Res. 2009;23(5):719-724.

43. Kujawski R, Bartkowiak Wieczorek J, Ozarowski M, et al. Curren knowledge on phytochemical profile of Epilobium sp. raw materials and extracts. Potential benefits in nutrition and phytotherapy of age-related diseases. Herba Pol. 2011;57:25-34.

44. Voynova E, Dimitrova S, Naydenova E, et al. Inhibitory action of extracts of Maclura aurantiaca and Epilobium hirsutum on tumour models in mice. Acta Physiol Pharmacol Bulg. 1991;17(4):50-52.

45. Hiermann A, Bucar F. Studies of Epilobium angustifolium extract on growth of accessory sexual organs in rats. J Ethnopharmacol. 1997;55(3): 179-183.

46. Vitalone A, Bordi F, Baldazzi C, et al. Anti-proliferative effect on a prostatic epithelial cell line (PZ-HPV-7) by Epilobium angustifolium L. Il Farmaco. 2001;56(5-7):483-489.

47. Vitalone A, Guizzetti M, Costa LG, et al. Extracts of various species of Epilobium inhibit proliferation of human prostate cells. J Pharm Pharmacol. 2003;55(5):683-690.

48. Lesuisse D, Berjonneau J, Ciot C, et al. Determination of oenothein B as the active $5-\alpha$-reductase-inhibiting principle of the folk medicine Epilobium parviflorum. J Nat Prod. 1996;59(5):490-492.

49. Ducrey B, Wolfender J, Marston A, et al. Analysis of flavonol glycosides of thirteen Epilobium species (Onagraceae) by LC-UV and thermospray LC-MS, Phytochemistry. Elsevier Science Ltd. 1995;38(1):129-137.

50. Wang CC, Chen LG, Yang LL. Antitumor activity of four macrocyclic ellagitannins from Cuphea hyssopifolia. Cancer Lett. 1999;140(12):195-200
51. Miyamoto K, Nomura M, Murayama T, et al. Antitumor activities of ellagitannins against sarcoma-180 in mice. Biol Pharm Bull. 1993;16(4):379-387.

52. Ducrey B, Marston A, Göhring S, et al. Inhibition of $5 \alpha$-reductase and aromatase by the ellagitannins oenothein A and oenothein B from Epilobium species. Planta Med. 1997;63(2):111-114.

53. Weijerman PC, Konig JJ, Wong ST, et al. Lipofection-mediated immortalization of human prostatic epithelial cells of normal and malignant origin using human papillomavirus type 18 DNA. Cancer Res. 1994;54(21):5579-5583.

54. Gupta S, Afaq F, Mukhtar H. Selective growth-inhibitory, cell-cycle deregulatory and apoptotic response of apigenin in normal versus human prostate carcinoma cells. Biochem Biophys Res Commun. 2001;287(4):914-920.

55. Horoszewicz JS, Leong SS, Kawinski E, et al. LNCaP model of human prostatic carcinoma. Cancer Res. 1983;43(4):1809-1818.

56. Kujawski R, Mrozikiewicz PM, Bogacz A, et al. Influence of standardized extract of Epilobium angustifolium on estrogen receptor alpha and beta expression in in vivo model. Ginekol Pol. 2010;81(8):600-605.

57. Kujawski R, Bartkowiak Wieczorek J, Karasiewicz M, et al. Influence of Epilobium angustifolium extract on $5 \alpha$-reductase type 2 and MAPK3 kinase gene expression in rats prostates. Herba Polonica. 2013;59(4):72-85

58. Coulson S, Rao A, Beck SL, et al. A phase II randomised double-blind placebo-controlled clinical trial investigating the efficacy and safety of ProstateEZE Max:a herbal medicine preparation for the management of symptoms of benign prostatic hypertrophy. Compl Ther Med. 2013;21(3):172-179

59. Sovak M, Seligson AL, Kucerova R, et al. Fluridil, a rationally designed topical agent for androgenetic alopecia:first clinical experience. Dermatol Surg. 2002;28(8):678-685.

60. Seligson AL, Campion BK, Brown JW, et al. Development of fluridil, a topical suppressor of the androgen receptor in androgenetic alopecia. Drug Dev Res. 2003;59:292-306.

61. Hajduch M, Sovak M, Kucerova R, et al. Current therapies of female androgenetic alopecia and use of fluridil, a novel topical antiandrogen. Scr Med (Brno). 2006;79(1):35-48.

62. Pierard Franchimont C, De Doncker P, Cauwenbergh G, et al. Ketoconazole shampoo:effect of long-term use in androgenic alopecia. Dermatology. 1998;196(4):474-477.

63. Jiang J, Tsuboi R, Kojima Y, et al. Topical application of ketoconazole stimulates hair growth in C3H/HeN mice. J Dermatol. 2005;32(4):243247

64. Keaney T. Emerging Therapies for Androgenetic Alopecia. J Drug Dermatol. 2015;14(9):1036-1040.

65. Jacobs JB, Monell C. Treatment of radiation-induced alopecia. Head Neck surg. 1979;2(2):154-159.

66. Rossi A, Anzalone A, Fortuna MC, et al. Multi-therapies in androgenetic alopecia:review and clinical experiences. Dermatol Ther. 2016;29(6):424-432

67. Fischer TW, Slominski A, Tobin DJ, et al. Melatonin and the hair follicle. J Pineal Res. 2008;44(1):1-15

68. Rimler A, Culig Z, Lupowitz Z, et al. Nuclear exclusion of the androgen receptor by melatonin. J Steroid Biochem Mol Biol. 2002;81(1):77-84.

69. Rimler A, Jockers R, Lupowitz Z, et al. Gi and RGS proteins provide biochemical control of androgen receptor nuclear exclusion. $J \mathrm{Mol} \mathrm{Neu}$ rosci. 2007;31(1):1-12. 
70. Fischer T, Burmeister G, Schmidt H, et al. Melatonin increases anagen hair rate in women with androgenetic alopecia or diffuse alopecia: results of a pilot randomized controlled trial. Br J Dermatol. 2004;150(2):341345 .

71. Charlesworth E, Kagey Sobotka A, Norman P, et al. Effect of cetirizine on mast cell-mediator release and cellular traffic during the cutaneous late-phase reaction. J Allergy Clin immunol. 1989;83(5):905-912.

72. Roch Arveiller M, Tissot M, Idohou N, et al. In vitro effect of cetirizine on PGE2 release by rat peritoneal macrophages and human monocytes. Agents Actions. 1994;43(1-2):13-16.

73. Traupe H, Happle R, Grobe H, et al. Polarization microscopy of hair in acrodermatitis enteropathica. Pediatr Dermatol. 1986;3(4):300-303.

74. Reichrath J, Schilli M, Kerber A, et al. Hair follicle expression of 1 , 25-dihydroxyvitamin D3 receptors during the murine hair cycle. $\mathrm{Br} J$ Dermatol. 1994;131(4):477-482.

75. Amor KT, Rashid RM, Mirmirani P. Does D matter? The role of vitamin $\mathrm{D}$ in hair disorders and hair follicle cycling. Dermatol Online J. 2010;16(2):p. 3 .

76. Chen G, Baechle A, Nevins TD, et al. Protection against cyclophosphamide-induced alopecia and inhibition of mammary tumor growth by topical 1, 25-dihydroxyvitamin D3 in mice. Int $J$ Cancer. 1998;75(2):303-309.

77. Wang J, Lu Z, Au JL S. Protection against chemotherapy-induced alopecia. Pharm Res. 2006;23(11):2505-2514.

78. Chasapis CT, Loutsidou AC, Spiliopoulou CA, et al. Zinc and human health: an update. Arch Toxicol. 2012;86(4):521-534.

79. Mc Call KA, Huang Cc, Fierke CA. Function and mechanism of zinc metalloenzymes. J Nutr. 2000;130(5S-Suppl):1437S-1446S.

80. Joly P. The use of methotrexate alone or in combination with low doses of oral corticosteroids in the treatment of alopecia totalis or universalis. J Am Acad Dermatol. 2006;55(4):632-636.
81. Vallee BL, Falchuk KH. The biochemical basis of zinc physiology. Physiol Rev. 1993;73(1):79-118.

82. Handjiski B, Eichmuller S, Hofmann U, et al. Alkaline phosphatase activity and localization during the murine hair cycle. $B J$ Dermatol. 1994;131(3):303-310.

83. Al Jaff A. Role of serum zinc and copper and zinc/copper ratio in alopecia areata. Iraqi J Pharm Sci. 2005;14:47-51.

84. Weismann K, Hoyer H. Zinc deficiency dermatoses. Etiology, clinical aspects and treatment. Hautarzt. 1982;33(8):405-410.

85. Cuscela D, Coffin D, Lupton GP, et al. Protection from radiation-induced alopecia with topical application of nitroxides:fractionated studies. Cancer J Sci Am. 1996;2(5); 273-278.

86. Hanson WR, Pelka AE, Nelson AK, et al. Subcutaneous or topical administration of 16,16-dimethyl prostaglandin E2 protects from radiation induced alopecia in mice. Int J Radiat Oncol Biol Phys. 1992;23(2):333337.

87. Severs GA, Griffin T, Werner Wasik M. Cicatricial alopecia secondary to radiation therapy:case report and review of the literature. Cutis. 2008;81(2):147-153.

88. Dupre A, Bonafe J, Carriere J. The hair in acrodermatitis interopathica-a disease indicator? Acta Derm Venereol. 1979;59(2):177-178.

89. Uno H, Zimbric ML, Albert DM, et al. Effect of latanoprost on hair growth in the bald scalp of the stump-tailed macacque:a pilot study. Act Derm Venereol. 2002;82(1):7-12.

90. Pierard Franchimont C, Goffin V, Henry F, et al. Nudging hair shedding by antidandruff shampoos. A comparison of $1 \%$ ketoconazole, $1 \%$ piroctone olamine and 1\% zinc pyrithione formulations. Int J Cosmet Sci. 2002;24(5):249-256. 